\title{
SNARE hypothesis 2000
}

A question that has kept scientists busy for some time is how specificity is achieved during membrane traffic. In other words, how does a vesicle distinguish, say, the plasma membrane from a lysosome? In 1993, Jim Rothman proposed a working model - the 'SNARE hypothesis' - and variations on this theme have dominated membrane fusion research ever since. The model has been seriously challenged over the past few years, but in the 15 September issue of Nature, the Rothman and Söllner laboratories present new evidence to support it.

According to the original hypothesis, transmembrane SNARE proteins present on the vesicle and target membranes pair up in antiparallel fashion to dock the membranes. The trans-SNARE complex consists of a syntaxin and a SNAP25 family member on the target membrane and a VAMP family member on the vesicle. The binding of two soluble proteins, NSF and SNAP, to the SNARE complex then drives membrane fusion. The specificity of SNARE pairing dictates the specificity of membrane recognition.

Many mechanistic predictions of the SNARE hypothesis have since been disproved. First, the function of NSF and SNAP is not to drive fusion but to open SNARE complexes to allow their recycling after fusion has occurred. Then, membrane docking can occur in the absence of SNAREs. Next, SNAREs are promiscuous in their interactions, at least in vitro. Last, the genome of the yeast Saccharomyces cerevisiae does not contain enough SNAREs to specify all the transport steps in the cell.

The Rothman and Söllner laboratories have now reconstituted yeast SNAREs into liposomes in all possible combinations and measured membrane mixing as a readout for correct pairing. They found remarkable specificity in SNARE pairing, as out of 33 possible combinations, only three known, one suspected and one novel SNARE combination occurred.
So, whereas SNAREs pair up almost randomly in solution, they are not at all promiscuous in the presence of lipid bilayers.

Rothman and colleagues also showed that SNAREs can only interact productively with their partners if they are in the correct orientation. So if a SNARE ends up on the wrong membrane after fusion has occurred, it won't be able to take part in a functional complex with its normal partners because it will be positioned upside down. Only after it has found its way back to its residence membrane can it form a fusogenic complex with its partners.

Using a similar approach, Söllner and colleagues found that SNARE complexes are made up of four SNAREs and not three. Indeed, the two $\alpha$-helices contributed by SNAP25 to the neuronal SNARE complex probably stem from two separate SNAREs in most other complexes. By using four SNAREs instead of three and by applying a combinatorial approach to SNARE pairing, there may be enough SNAREs after all to allow each SNARE complex to act in only one transport step.

So, although parts of the SNARE hypothesis have been disproved, its core postulate seems to have survived the test of time - formation of SNARE complexes confers specificity to membrane fusion. The regulation of SNARE-mediated membrane fusion by Rab proteins, tethering factors and proteins of the Munc family should now keep us busy for the next few years.

\section{(1) References and links} ORIGINAL RESEARCH PAPERS McNew, J. A. et al. Compartmental specificity of cellular membrane fusion encoded in SNARE proteins Nature 407, 153-159 (2000) | Parlati, F. et al. Topological restriction of SNARE-dependent membrane fusion. Nature 407, 194-198 (2000) Fukuda, R et al. Functional architecture of an intracellular membrane t-SNARE. Nature $\mathbf{4 0 7}$ 198-202 (2000)

NEWS AND Views Scales, S. J. et al. The specifics of membrane fusion. Nature $\mathbf{4 0 7}$ 144-146 (2000)

REVIEW Jahn, R. \& Südhof, T C. Membran fusion and exocytosis. Annu. Rev. Biochem. 68 863-911 (1999)

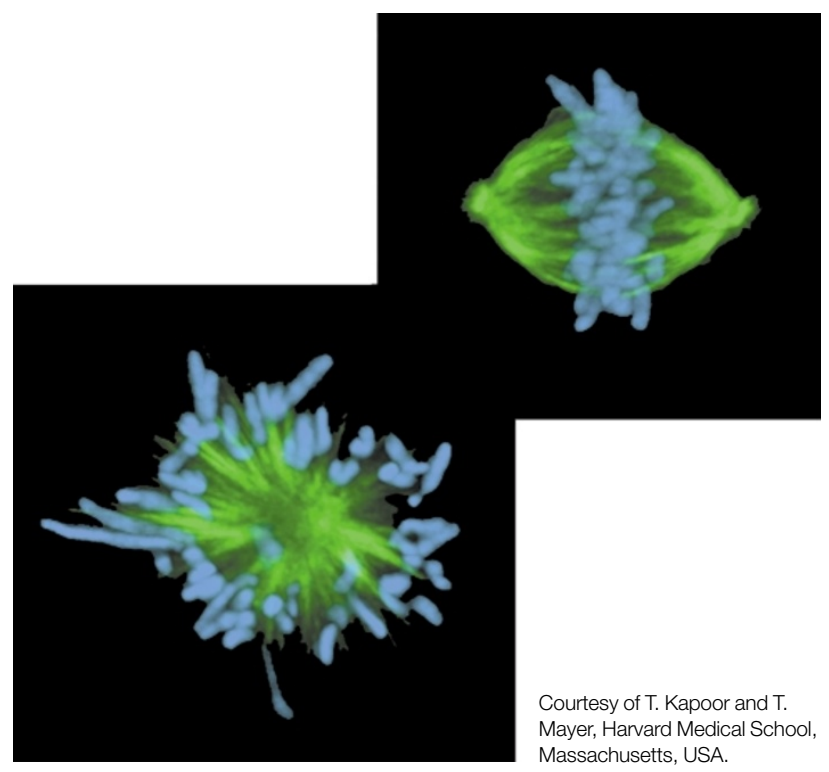

CELL DIVISION

\section{Another star on drugs}

The function of the microtubule spindle during mitosis is to distribute replicated DNA equally between daughter cells. Last year Mitchison and colleagues discovered a new drug that interferes with spindle formation and they now show that this drug can be useful for the study of spindle dynamics.

Monastrol is a small, membrane-permeant compound whose action is rapidly reversible. This makes it a perfect tool for the study of mitotic spindles. Instead of targeting microtubules like many other drugs, monastrol specifically inhibits the motility of the mitotic kinesin Eg5, resulting in the formation of monopolar spindles, also called monoasters.

Kapoor et al. found that, in the presence of monastrol, centrosomes duplicate but do not separate. If the drug is added to preformed spindles in a cell-free assay, the two poles move towards each other until they merge. This confirms that Eg5 functions in centrosome separation and in the maintenance of microtubule crosslinking at the spindle midzone.

Moreover, Kapoor et al. made two interesting observations using monastrol. First, they discovered forces orthogonal to the spindle axis that had previously not been recognized.

They also found that the spindle checkpoint protein Mad2 is probably sensitive to the exact number of microtubules at each kinetochore, tension at each kinetochore or the dynamic status of the kinetochore. These observations now need to be followed up.

So monastrol has been validated as a tool to study spindle dynamics, and has opened two new avenues for research.

Raluca Gagescu

(9) References and links

ORIGINAL RESEARCH PAPER Kapoor, T. M. et al. Probing spindle assembly mechanisms with monastrol, a small molecule inhibitor of the mitotic kinesin Eg5. J. Cell Biol. 150, 975-988 (2000)

FURTHER READING Mayer, T. U. et al. Small molecule inhibitor of mitotic spindle bipolarity identified in a phenotype-based screen. Science 286, 971-974 (1999) 